\title{
An Improved EPMA Method for Gaining High Precision and High Accuracy Determination of Trace Elements in Quartz
}

\author{
Shui-Yuan Yang ${ }^{1}$, Ji-Qiang Cui ${ }^{1}$, Shao-Yong Jiang ${ }^{1,2}$, Jing Xie ${ }^{3}$ \\ 1. State Key Laboratory of Geological Processes and Mineral Resources, China University of \\ Geosciences, Wuhan, PR China \\ 2. Faculty of Earth Resources and Collaborative Innovation Center for Scarce and Strategic Mineral \\ Resources, China University of Geosciences, Wuhan, PR China \\ 3. Key Laboratory of Continental Collision and Plateau Uplift, Institute of Tibetan Plateau Research, \\ Chinese Academy of Sciences, Beijing, PR China
}

It is a challenge to obtain high precision and high accuracy data of trace elements in minerals using electron probe microanalysis (EPMA) method. In this study, we investigate the factors which affect the precision and accuracy for trace element analysis of $\mathrm{Al}$ and Ti in quartz, using the conclusions of previous literatures and the experiments from a JEOL JXA-8100 EPMA at the State Key Laboratory of Geological Processes and Mineral Resources, China University of Geosciences (Wuhan). Our aim is to gain an analytical method that can improve the precision and accuracy of these measurements.

In EPMA trace element measurements, the characteristic X-ray intensity is typically enhanced by changing the acceleration voltage, increasing the counting time, or increasing the beam current (e.g. [1, 2]). The detection limit (DL) is in reverse proportion to the square root of the beam current and the counting time. With an increase in the accelerating voltage, the detection limit of $\mathrm{Al}$ in quartz first decreases slightly and then increases slightly, while the detection limit of Ti decreases [3]. Consequently, it is ideal to choose a higher accelerating voltage, such as $20 \mathrm{kV}$ or $25 \mathrm{kV}$, to effectively improve the analytical precision and lower the detection limit of $\mathrm{Al}$ and $\mathrm{Ti}$ in quartz. In addition, the simultaneous use of multiple crystals for single elements can improve the analytical precision [1]. The precision of trace element analyses can be improved by all four approaches.

The main factors affecting the analytical accuracy of $\mathrm{Al}$ and $\mathrm{Ti}$ in quartz include the processing of interferences, the methods used to determine the background and fragility of quartz lattices at high beam currents (e.g. $[1,3,4])$.

Small interferences can cause large errors in the analysis of trace element by EPMA. Therefore, some key aspects relating to interference for the analysis of $\mathrm{Al}$ and $\mathrm{Ti}$ in quartz must be considered [3]. Firstly, the interference peaks can be found in the background and it is necessary to avoid the potential background interference peak when choosing background positions. Secondly, some neighboring phases that contain $\mathrm{Al}$ and $\mathrm{Ti}$ will produce an apparent secondary fluorescence signal in the detector and thus it is necessary to maintain a safe distance from the interference mineral or correct for the interference (e.g. [5-7]). Thirdly, a "step" background always occurs at a position of 82-83 $\mathrm{mm}$ on PETJ spectrometers, and a safe distance from the "step" have to keep when choosing background positions. Finally, a "hole" in the background sometimes occur both adjacent to and directly underneath the Ti-K $\alpha$ X-ray peak position in quartz, a "blank" correction method [1] can be used to correct for this systematic inaccuracy.

Detailed wavelength scanning indicates that the background of $\mathrm{Al}$ in quartz is a concave curve and the classical linear regression between two-point selected at background will produce incorrect results. The 
detection accuracy can be improved greatly by using the multipoint background method to fit the background curve equation and calculate the background value. For the $\mathrm{Ti}$ in quartz, the background is an approximately straight line, and both the two-point background method and the multipoint background method can obtain accurate results.

It is very easy for the crystal structure of quartz to be destroyed at high beam currents, resulting in the migration of elements and inaccurate analytical results (e.g. [4, 8]). A detailed time dependent intensity (TDI) study on the peaks of Al-K $\alpha$ and Ti-K $\alpha$ and both sides of these peaks at high beam currents (500 $\mathrm{nA}$ ) indicated that the peaks of $\mathrm{Al}-\mathrm{K} \alpha$ and $\mathrm{Ti}-\mathrm{K} \alpha$ and their backgrounds were found to show variations in TDI at high beam currents and small beam diameters [3]. Because the TDI variation is different at different background positions and the count changes in a short timescale, subsection determination [1] cannot completely eliminate the influence of TDI variation. In addition, the signal intensity of Al-K $\alpha$ and Ti-K $\alpha$ initially changes over a very short period when the beam diameter is small (Figure 1). The $20 \mu \mathrm{m}$ quadratic polynomial curves for both $\mathrm{Al}-\mathrm{K} \alpha$ and $\mathrm{Ti}-\mathrm{K} \alpha$ are approximately horizontal. The intensity value of the 5 $\mu \mathrm{m}$ quadratic polynomial curve at time zero is equal to that of $20 \mu \mathrm{m}$ quadratic polynomial curve. However, the intensity value of the $1 \mu \mathrm{m}$ quadratic polynomial curve at time zero is different from that of $20 \mu \mathrm{m}$ quadratic polynomial curve. Consequently, it is necessary to select a large beam diameter (up to $20 \mu \mathrm{m}$ ) to avoid TDI variations at high currents, or a medium beam diameter with TDI correction to obtain accurate results, and TDI correction method can't get the accurate results when the beam diameter is extremely small [9].

\section{References:}

[1] J. J. Donovan et al, Am. Miner. 96 (2011) p. 274.

[2] V. G. Batanova et al, Chem. Geol. 419 (2015) p. 149.

[3] J. Q. Cui et al, Microsc. Microanal. In Press, DOI: $10.1017 /$ S1431927618015672

[4] A. Müller et al, Eur. J. Mineral. 15 (2003) p. 747.

[5] M. J. Jercinovic and M. L. Williams, Am. Miner. 90 (2005) p. 526.

[6] J. Fournelle, Microsc. Microanal. 13 (2007) p. 1390.

[7] A. Y. Borisova et al, Chem. Geol. 490 (2018) p. 22.

[8] A. M. van den Kerkhof et al, Contrib. Mineral. Petrol. 146 (2004) p. 637.

[9] This work was supported by the National Key R\&D Plan of China (No. 2017YFC0601404) and the National Natural Science Foundation of China (No. 41773040).
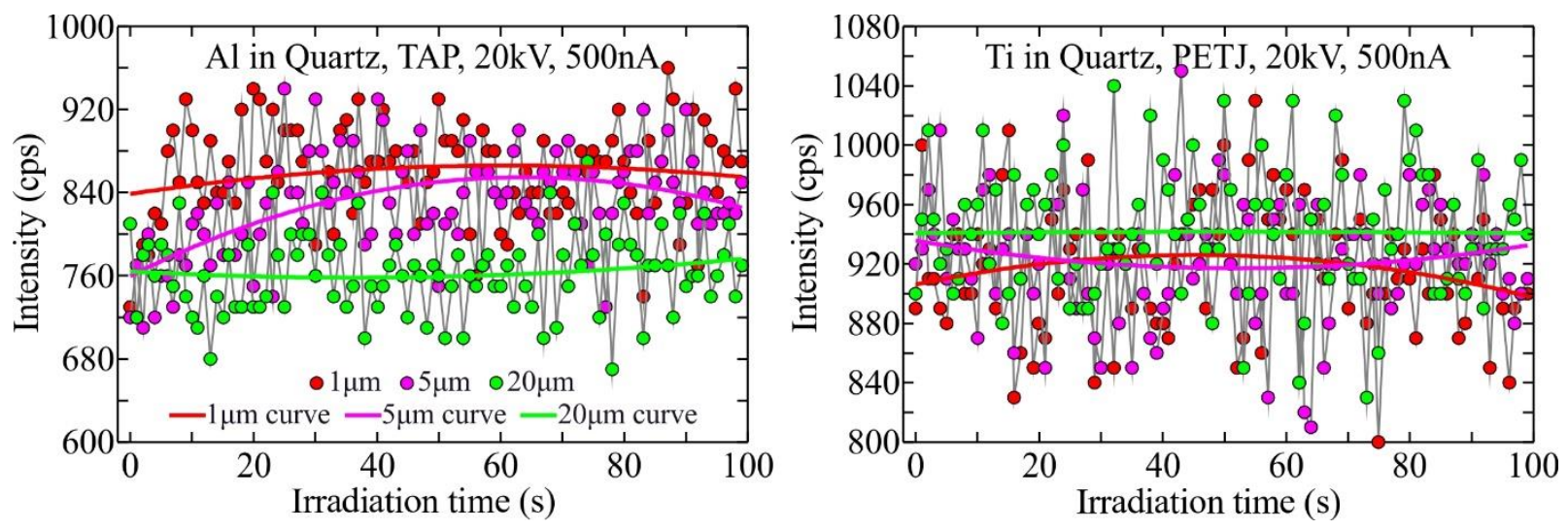

Figure 1. Al-K $\alpha$ (left) and Ti-K $\alpha$ (right) counts as a function of beam diameter and time at the condition of $20 \mathrm{kV}$ and $500 \mathrm{nA}$. The quadratic polynomial curves are also showed in figure. 